\title{
CENTRAL SCHEMES AND CONTACT DISCONTINUITIES
}

\author{
Alexander Kurganov ${ }^{1}$ and Guergana Petrova ${ }^{1}$
}

\begin{abstract}
We introduce a family of new second-order Godunov-type central schemes for one-dimensional systems of conservation laws. They are a less dissipative generalization of the central-upwind schemes, proposed in [A. Kurganov et al., submitted to SIAM J. Sci. Comput.], whose construction is based on the maximal one-sided local speeds of propagation. We also present a recipe, which helps to improve the resolution of contact waves. This is achieved by using the partial characteristic decomposition, suggested by Nessyahu and Tadmor [J. Comput. Phys. 87 (1990) 408-463], which is efficiently applied in the context of the new schemes. The method is tested on the one-dimensional Euler equations, subject to different initial data, and the results are compared to the numerical solutions, computed by other second-order central schemes. The numerical experiments clearly illustrate the advantages of the proposed technique.
\end{abstract}

Mathematics Subject Classification. 65M10, 65M05.

Received: May 29, 2000. Revised: October 2, 2000.

\section{INTRODUCTION}

In this paper, we study second-order Godunov-type central schemes for one-dimensional systems of hyperbolic conservation laws

$$
u_{t}+f(u)_{x}=0,
$$

subject to the initial data

$$
u(x, 0)=u_{0}(x) .
$$

Here, $u(x, t):=\left(u_{1}(x, t), \ldots, u_{N}(x, t)\right)$ is the unknown vector-function and $f(u):=\left(f_{1}(u), \ldots, f_{N}(u)\right)$ is the flux vector with the Jacobian $A:=\left(\frac{\partial f_{i}}{\partial u_{j}}\right), i, j=1, \ldots, N$.

A first-order stable central scheme was first introduced by Lax and Friedrichs in [5,15]. Unfortunately, the Lax-Friedrichs (LxF) scheme has a large numerical dissipation which leads to a poor resolution of the nonsmooth parts of the solution (unless a huge number of grid points is used, which may be practically impossible).

Keywords and phrases. Euler equations of gas dynamics, partial characteristic decomposition, fully-discrete and semi-discrete central schemes.

${ }^{1}$ Department of Mathematics, University of Michigan, Ann Arbor, MI 48109-1109, USA. e-mail: kurganov@math.1sa.umich.edu, petrova@math.1sa.umich.edu 
In [22], Nessyahu and Tadmor proposed a second-order generalization (the NT scheme) of the LxF scheme. The NT scheme has smaller numerical viscosity and resolves shock waves, rarefactions and contact discontinuities much better than the first-order LxF scheme. Higher-order generalizations of the NT scheme were introduced in $[3,17,21]$. We refer the reader to $[24,25]$ for an alternative staggered approach, and to $[1,2,9,18,19]$ for examples of central schemes in the multidimensional case.

A disadvantage of the staggered central schemes is that their numerical viscosity is proportional to $(\Delta x)^{2 r} / \Delta t$, where $r$ is the formal order of the scheme. This results in excessive numerical dissipation if a small time step is enforced or a long-time integration is performed. To overcome this difficulty, a new family of nonstaggered fully- and semi-discrete central schemes has been recently introduced in [11-14]. The key idea behind their construction is to obtain a more precise estimate of the width of the local Riemann fans by utilizing the maximal local speeds of propagation. This reduces the numerical viscosity to $\mathcal{O}(\Delta t)^{2 r-1}$, and therefore makes it possible to efficiently use central schemes when the time step is small.

Even though the numerical dissipation of the aforementioned high-order central schemes is small, it is not small enough to provide a satisfactory resolution of contact discontinuities. A possible way to improve the resolution was proposed in [22]. The main idea is to isolate the less expensive characteristic projection on the linear contact field, and to use the component-wise approach for the other, nonlinear fields (see Sect. 4 in [22]). The effect of this partial characteristic decomposition can be amplified if it is used together with the artificial compression method (ACM), applied to the isolated contact field (see $[6,7,22])$. The above technique was extended in [20] in the multidimensional case. However, the drawback of using the ACM is that it requires a certain a priori information about the solution in order to determine the optimal amount of the added "anti-diffusion".

The main purpose of this paper is to present (in the context of nonstaggered schemes from $[13,14]$ ) an alternative use of the partial characteristic information. First, in Section 2, we derive a new second-order fully-discrete scheme and then, in Section 3, passing to the limit as $\Delta t \rightarrow 0$, we obtain a new second-order semi-discrete scheme. Compared with the schemes from [13,14], the proposed schemes have smaller numerical viscosity, which leads to a sharper resolution of contact discontinuities.

In Section 4, we use the partial characteristic decomposition to treat the linear and nonlinear fields separately, and this helps to improve the resolution of the contact waves. At the same time, the numerical examples, presented in Section 5 , clearly demonstrate that the reduced numerical dissipation does not influence the stability of the proposed central schemes. We would also like to mention that the computational cost of the partial decomposition, used in the construction of the new schemes, is relatively low. This preserves one of the main advantages of the central schemes - their efficiency.

\section{Fully-Discrete SCHEME}

In this section, we derive a modification of the second-order fully-discrete central-upwind scheme, introduced in [13]. First, we would like to recall the construction of central-upwind schemes (see [13]), which consist of three consecutive steps - reconstruction, evolution and projection back onto the original grid.

We will consider only uniform grids and use the following notation, $x_{j}:=j \Delta x, x_{j \pm \frac{1}{2}}:=(j \pm 1 / 2) \Delta x$, $t^{n}:=n \Delta t, \lambda:=\Delta t / \Delta x, u_{j}^{n} \approx u\left(x_{j}, t^{n}\right)$, and

$$
\bar{u}_{j}^{n} \approx \bar{u}\left(x_{j}, t^{n}\right):=\frac{1}{\Delta x} \int_{x_{j-\frac{1}{2}}}^{x_{j+\frac{1}{2}}} u\left(\xi, t^{n}\right) \mathrm{d} \xi .
$$

Here, $\Delta x$ and $\Delta t$ are small spatial and time scales, respectively, and $\bar{u}_{j}^{n}$ are the computed cell averages at time level $t=t^{n}$. 


\section{Reconstruction}

Assume that at time level $t=t^{n}$ we have computed the cell averages $\left\{\bar{u}_{j}^{n}\right\}$. We then reconstruct the second-order piecewise linear interpolant

$$
\widetilde{u}\left(x, t^{n}\right)=l_{j}^{n}(x):=\bar{u}_{j}^{n}+s_{j}^{n}\left(x-x_{j}\right), \quad x_{j-\frac{1}{2}}<x<x_{j+\frac{1}{2}}, \quad \forall j .
$$

To ensure the non-oscillatory nature of this reconstruction, the slopes $\left\{s_{j}^{n}\right\}$ should be computed using a nonlinear limiter. A wide variety of such limiters can be found, for example, in [7,8,10,16,22, 23]. In the numerical experiments, presented below, we use the so-called minmod limiter [7,16,23]

$$
s_{j}^{n}=\operatorname{minmod}\left(\theta \frac{\bar{u}_{j}^{n}-\bar{u}_{j-1}^{n}}{\Delta x}, \frac{\bar{u}_{j+1}^{n}-\bar{u}_{j-1}^{n}}{2 \Delta x}, \theta \frac{\bar{u}_{j+1}^{n}-\bar{u}_{j}^{n}}{\Delta x}\right), \quad 1 \leq \theta \leq 2,
$$

where

$$
\operatorname{minmod}\left(x_{1}, x_{2}, \ldots\right):=\left\{\begin{array}{lc}
\min _{j}\left\{x_{j}\right\}, & \text { if } x_{j}>0 \forall j \\
\max _{j}\left\{x_{j}\right\}, & \text { if } x_{j}<0 \forall j \\
0, & \text { otherwise. }
\end{array}\right.
$$

Notice that larger $\theta$ 's in (2.2) correspond to a less dissipative, but still non-oscillatory reconstruction (2.1). Our experience shows that in practice the optimal value of $\theta$ for the Euler system of gas dynamics is about $\theta=1.3$.

\section{Evolution}

The interpolant (2.1) may be discontinuous at the interface points $\left\{x_{j+\frac{1}{2}}\right\}$. These discontinuities propagate with right- and left-sided local speeds, which can be estimated by

$$
a_{j+\frac{1}{2}}^{+}:=\max _{\omega \in C\left(u_{j+\frac{1}{2}}^{-}, u_{j+\frac{1}{2}}^{+}\right)}\left\{\lambda_{N}(A(\omega)), 0\right\},
$$

and

$$
a_{j+\frac{1}{2}}^{-}:=\min _{\omega \in C\left(u_{j+\frac{1}{2}}^{-}, u_{j+\frac{1}{2}}^{+}\right)}\left\{\lambda_{1}(A(\omega)), 0\right\}
$$

respectively. Here, $\lambda_{1}<\ldots<\lambda_{N}$ are the $N$ eigenvalues of the Jacobian $A$, and $C\left(u_{j+\frac{1}{2}}^{-}, u_{j+\frac{1}{2}}^{+}\right)$is the Riemann admissible curve in the phase space that connects the left, $u_{j+\frac{1}{2}}^{-}:=l_{j}^{n}\left(x_{j+\frac{1}{2}}\right)$, and the right, $u_{j+\frac{1}{2}}^{+}:=l_{j+1}^{n}\left(x_{j+\frac{1}{2}}\right)$, states. For example, in the genuinely nonlinear or linearly degenerate case, we have

$$
\begin{aligned}
a_{j+\frac{1}{2}}^{+} & =\max \left\{\lambda_{N}\left(A\left(u_{j+\frac{1}{2}}^{-}\right)\right), \lambda_{N}\left(A\left(u_{j+\frac{1}{2}}^{+}\right)\right), 0\right\}, \\
a_{j+\frac{1}{2}}^{-} & =\min \left\{\lambda_{1}\left(A\left(u_{j+\frac{1}{2}}^{-}\right)\right), \lambda_{1}\left(A\left(u_{j+\frac{1}{2}}^{+}\right)\right), 0\right\} .
\end{aligned}
$$

Next, let us denote by $x_{j+\frac{1}{2}, l}^{n}:=x_{j+\frac{1}{2}}+\Delta t a_{j+\frac{1}{2}}^{-}$and $x_{j+\frac{1}{2}, r}^{n}:=x_{j+\frac{1}{2}}+\Delta t a_{j+\frac{1}{2}}^{+}$and consider the non-equal rectangular domains (see Fig. 2.1),

$$
\left[x_{j-\frac{1}{2}, r}^{n}, x_{j+\frac{1}{2}, l}^{n}\right] \times\left[t^{n}, t^{n+1}\right] \quad \text { and } \quad\left[x_{j+\frac{1}{2}, l}^{n}, x_{j+\frac{1}{2}, r}^{n}\right] \times\left[t^{n}, t^{n+1}\right]
$$




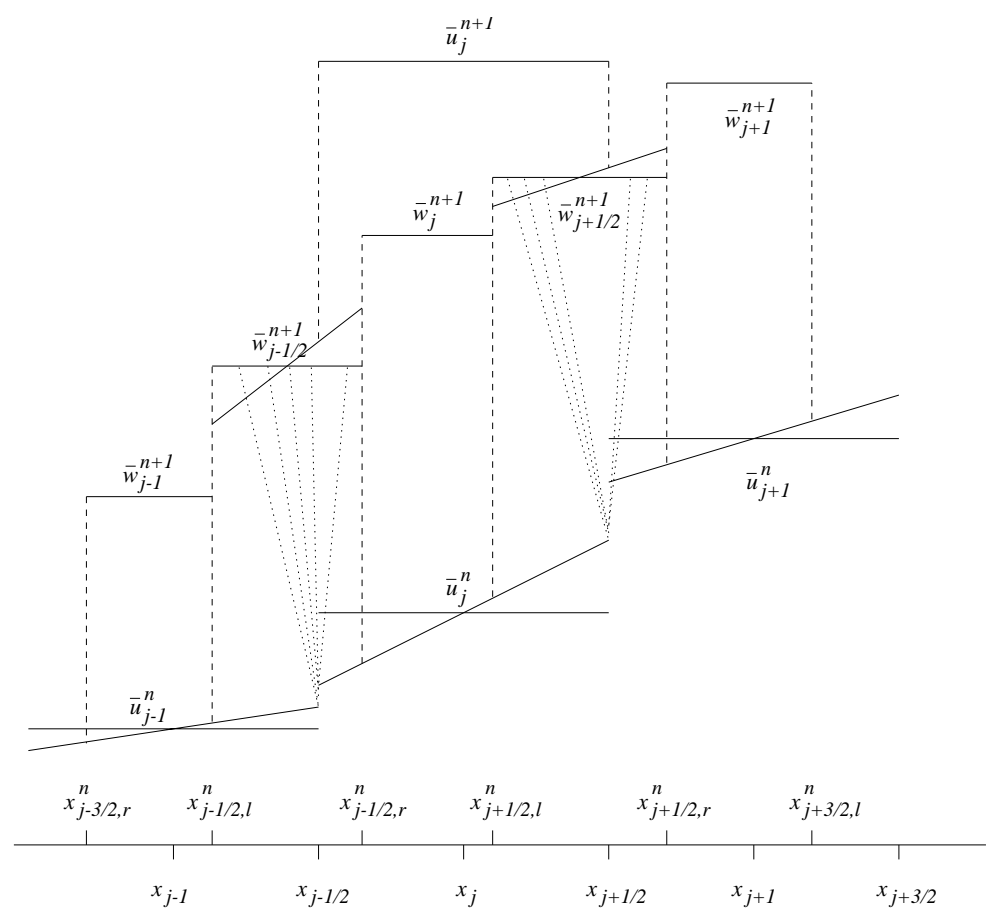

Figure 2.1. Central-upwind differencing.

where the solution of (1.1) with the initial data $\widetilde{u}\left(x, t^{n}\right)$ is smooth and nonsmooth, respectively.

Integration of (1.1) over these domains together with the midpoint approximation of the flux integrals, results in the following intermediate cell averages, outlined in Figure 2.1,

$$
\begin{aligned}
\bar{w}_{j}^{n+1} & =\frac{1}{x_{j+\frac{1}{2}, l}^{n}-x_{j-\frac{1}{2}, r}^{n}}\left[\int_{x_{j-\frac{1}{2}, r}^{n}}^{x_{j+\frac{1}{2}, l}^{n}} \tilde{u}\left(x, t^{n}\right) \mathrm{d} x-\int_{t^{n}}^{t^{n+1}}\left(f\left(u\left(x_{j+\frac{1}{2}, l}^{n}, t\right)\right)-f\left(u\left(x_{j-\frac{1}{2}, r}^{n}, t\right)\right)\right) \mathrm{d} t\right] \\
& =\bar{u}_{j}^{n}+\frac{\Delta t}{2}\left(a_{j-\frac{1}{2}}^{+}+a_{j+\frac{1}{2}}^{-}\right) s_{j}^{n}-\frac{\lambda}{1+\lambda\left(a_{j+\frac{1}{2}}^{-}-a_{j-\frac{1}{2}}^{+}\right)}\left[f\left(u_{j+\frac{1}{2}, l}^{n+\frac{1}{2}}\right)-f\left(u_{j-\frac{1}{2}, r}^{n+\frac{1}{2}}\right)\right],
\end{aligned}
$$

and

$$
\begin{aligned}
\bar{w}_{j+\frac{1}{2}}^{n+1}= & \frac{1}{x_{j+\frac{1}{2}, r}^{n}-x_{j+\frac{1}{2}, l}^{n}}\left[\int_{x_{j+\frac{1}{2}, l}^{n}}^{x_{j+\frac{1}{2}, r}^{n}} \tilde{u}\left(x, t^{n}\right) \mathrm{d} x-\int_{t^{n}}^{t^{n+1}}\left(f\left(u\left(x_{j+\frac{1}{2}, r}^{n}, t\right)\right)-f\left(u\left(x_{j+\frac{1}{2}, l}^{n}, t\right)\right)\right) \mathrm{d} t\right] \\
= & \frac{a_{j+\frac{1}{2}}^{+} \bar{u}_{j+1}^{n}-a_{j+\frac{1}{2}}^{-} \bar{u}_{j}^{n}}{a_{j+\frac{1}{2}}^{+}-a_{j+\frac{1}{2}}^{-}}-\frac{\left(\Delta x+a_{j+\frac{1}{2}}^{-} \Delta t\right) a_{j+\frac{1}{2}}^{-} s_{j}^{n}}{2\left(a_{j+\frac{1}{2}}^{+}-a_{j+\frac{1}{2}}^{-}\right)}-\frac{\left(\Delta x-a_{j+\frac{1}{2}}^{+} \Delta t\right) a_{j+\frac{1}{2}}^{+} s_{j+1}^{n}}{2\left(a_{j+\frac{1}{2}}^{+}-a_{j+\frac{1}{2}}^{-}\right)} \\
& -\frac{1}{a_{j+\frac{1}{2}}^{+}-a_{j+\frac{1}{2}}^{-}}\left[f\left(u_{j+\frac{1}{2}, r}^{n+\frac{1}{2}}\right)-f\left(u_{j+\frac{1}{2}, l}^{n+\frac{1}{2}}\right)\right] .
\end{aligned}
$$


Here, the midpoint values are obtained from the corresponding Taylor expansions,

$$
\begin{array}{ll}
u_{j+\frac{1}{2}, l}^{n+\frac{1}{2}}:=u_{j+\frac{1}{2}, l}^{n}-\frac{\Delta t}{2} f\left(u_{j+\frac{1}{2}, l}^{n}\right)_{x}, & u_{j+\frac{1}{2}, l}^{n}:=\bar{u}_{j}^{n}+\Delta x s_{j}^{n}\left(\lambda a_{j+\frac{1}{2}}^{-}+\frac{1}{2}\right), \\
u_{j+\frac{1}{2}, r}^{n+\frac{1}{2}}:=u_{j+\frac{1}{2}, r}^{n}-\frac{\Delta t}{2} f\left(u_{j+\frac{1}{2}, r}^{n}\right)_{x}, & u_{j+\frac{1}{2}, r}^{n}:=\bar{u}_{j+1}^{n}+\Delta x s_{j+1}^{n}\left(\lambda a_{j+\frac{1}{2}}^{+}-\frac{1}{2}\right) .
\end{array}
$$

Note that one can avoid the computation of the Jacobian in (2.7) by using a component-wise evaluation of $f_{x}$, consult $[22]$.

\section{Projection}

At the final step we take the piecewise linear interpolant

$$
\widetilde{w}\left(x, t^{n+1}\right):=\sum_{j}\left\{\left[\bar{w}_{j+\frac{1}{2}}^{n+1}+s_{j+\frac{1}{2}}^{n+1}\left(x-\frac{x_{j+\frac{1}{2}, l}^{n}+x_{j+\frac{1}{2}, r}^{n}}{2}\right)\right] \chi_{\left[x_{j+\frac{1}{2}, l}^{n}, x_{j+\frac{1}{2}, r}^{n}\right]}+\bar{w}_{j}^{n+1} \chi_{\left[x_{j-\frac{1}{2}, r}^{n}, x_{j+\frac{1}{2}, l}^{n}\right.}\right\},
$$

reconstructed from the evolved intermediate cell averages (2.5)-(2.6), and project it back onto the original grid by averaging $(2.8)$ over $\left[x_{j-\frac{1}{2}}, x_{j+\frac{1}{2}}\right]$. Notice, that in $(2.8)$ we do not need a linear reconstruction over the interval $\left[x_{j-\frac{1}{2}, r}^{n}, x_{j+\frac{1}{2}, l}^{n}\right]$, since it will be averaged out by the projection (see Fig. 2.1). This results in

$$
\begin{aligned}
\bar{u}_{j}^{n+1}=\frac{1}{\Delta x} \int_{x_{j-\frac{1}{2}}}^{x_{j+\frac{1}{2}}} \widetilde{w}\left(\xi, t^{n+1}\right) \mathrm{d} \xi= & \lambda a_{j-\frac{1}{2}}^{+} \bar{w}_{j-\frac{1}{2}}^{n+1}+\left[1+\lambda\left(a_{j+\frac{1}{2}}^{-}-a_{j+\frac{1}{2}}^{+}\right)\right] \bar{w}_{j}^{n+1}-\lambda a_{j+\frac{1}{2}}^{-} \bar{w}_{j+\frac{1}{2}}^{n+1} \\
& +\frac{\lambda \Delta t}{2}\left[s_{j+\frac{1}{2}}^{n+1} a_{j+\frac{1}{2}}^{+} a_{j+\frac{1}{2}}^{-}-s_{j-\frac{1}{2}}^{n+1} a_{j-\frac{1}{2}}^{+} a_{j-\frac{1}{2}}^{-}\right] .
\end{aligned}
$$

This completes the construction of the second-order central-upwind fully-discrete scheme: $(2.2)-(2.7),(2.9)$.

The key issue, which leaves us an additional degree of freedom, is the choice of the slopes $\left\{s_{j+\frac{1}{2}}^{n+1}\right\}$. This can be done in different ways. For example, following the component-wise approach from [14], one may take

$$
s_{j+\frac{1}{2}}^{n+1}=2 \cdot \operatorname{minmod}\left(\theta \frac{\bar{w}_{j+\frac{1}{2}}^{n+1}-\bar{w}_{j}^{n+1}}{x_{j+\frac{1}{2}, r}^{n}-x_{j-\frac{1}{2}, r}^{n}}, \frac{\bar{w}_{j+1}^{n+1}-\bar{w}_{j}^{n+1}}{x_{j+\frac{3}{2}, l}^{n}+x_{j+\frac{1}{2}, r}^{n}-x_{j+\frac{1}{2}, l}^{n}-x_{j-\frac{1}{2}, r}^{n}}, \theta \frac{\bar{w}_{j+1}^{n+1}-\bar{w}_{j+\frac{1}{2}}^{n+1}}{x_{j+\frac{3}{2}, l}^{n}-x_{j+\frac{1}{2}, l}^{n}}\right) .
$$

Here, the distances

$$
x_{j+\frac{1}{2}, r}^{n}-x_{j-\frac{1}{2}, r}^{n}=\Delta x+\Delta t\left(a_{j+\frac{1}{2}}^{+}-a_{j-\frac{1}{2}}^{+}\right) \quad \text { and } \quad x_{j+\frac{3}{2}, l}^{n}-x_{j+\frac{1}{2}, l}^{n}=\Delta x+\Delta t\left(a_{j+\frac{3}{2}}^{-}-a_{j+\frac{1}{2}}^{-}\right),
$$

are of order $\Delta x$ for small $\Delta t$, and thus the slopes $s_{j+\frac{1}{2}}^{n+1}$ are proportional to $\Delta w / \Delta x$ as $\Delta t \sim 0$. Another choice of $s_{j+\frac{1}{2}}^{n+1}$ is proposed in $(4.2)$, where the slopes are $\sim \Delta w / \Delta t$. As it can be seen in Figure 2.2 , both reconstructions are non-oscillatory, but the first one leads to an excessive numerical dissipation, which is amplified when we pass to a semi-discrete limit as $\Delta t \rightarrow 0$ (see Sects. 3 and 4 ).

However, the amount of numerical viscosity in the scheme (2.2)-(2.7), (2.9) can be decreased if a partial characteristic information is employed in the computation of the slopes $s_{j+\frac{1}{2}}^{n+1}$, as described in Section 4 . Such an 


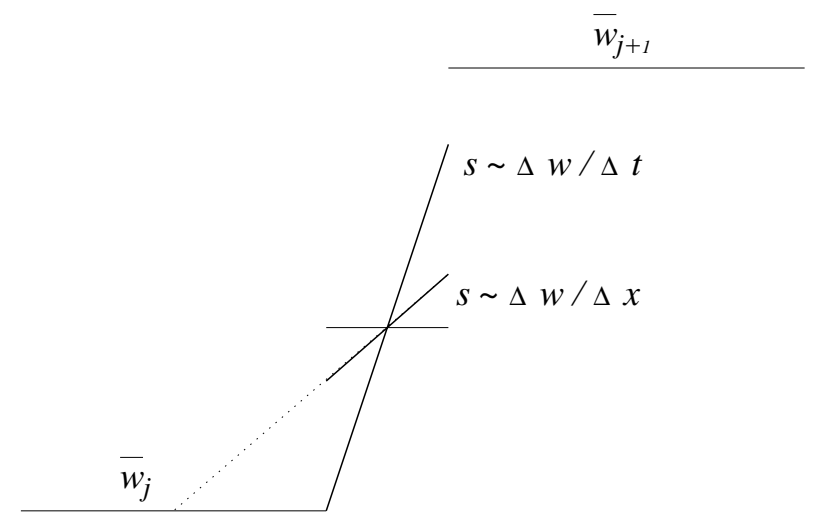

Figure 2.2. Two possible choices of $s_{j+\frac{1}{2}}^{n+1}-$ using (2.10) or (4.2).

approach leads to a sharper resolution of contact discontinuities, and this can be seen in the numerical examples presented in Section 5.

\section{Semi-Discrete sCheme}

In this section, we derive a family of semi-discrete central-upwind schemes for (1.1), proceeding along the lines of $[13,14]$. From $(2.9)$ we obtain that

$$
\begin{aligned}
\frac{\mathrm{d}}{\mathrm{d} t} \bar{u}_{j}(t)= & \lim _{\Delta t \rightarrow 0} \frac{\bar{u}_{j}^{n+1}-\bar{u}_{j}^{n}}{\Delta t}=\frac{a_{j-\frac{1}{2}}^{+}}{\Delta x} \lim _{\Delta t \rightarrow 0} \bar{w}_{j-\frac{1}{2}}^{n+1}+\lim _{\Delta t \rightarrow 0} \frac{1}{\Delta t}\left\{\left[1+\lambda\left(a_{j+\frac{1}{2}}^{-}-a_{j+\frac{1}{2}}^{+}\right)\right] \bar{w}_{j}^{n+1}-\bar{u}_{j}^{n}\right\} \\
& -\frac{a_{j+\frac{1}{2}}^{-}}{\Delta x} \lim _{\Delta t \rightarrow 0} \bar{w}_{j+\frac{1}{2}}^{n+1}+\frac{1}{2 \Delta x} \lim _{\Delta t \rightarrow 0}\left(\Delta t s_{j+\frac{1}{2}}^{n+1} a_{j+\frac{1}{2}}^{+} a_{j+\frac{1}{2}}^{-}-\Delta t s_{j-\frac{1}{2}}^{n+1} a_{j-\frac{1}{2}}^{+} a_{j-\frac{1}{2}}^{-}\right) .
\end{aligned}
$$

The first three limits on the RHS of (3.1) can be computed as follows. From (2.5) and (2.6) we obtain

$$
\lim _{\Delta t \rightarrow 0} \frac{1}{\Delta t}\left\{\left[1+\lambda\left(a_{j+\frac{1}{2}}^{-}-a_{j+\frac{1}{2}}^{+}\right)\right] \bar{w}_{j}^{n+1}-\bar{u}_{j}^{n}\right\}=\frac{a_{j+\frac{1}{2}}^{-} u_{j+\frac{1}{2}}^{-}-a_{j+\frac{1}{2}}^{+} u_{j+\frac{1}{2}}^{+}}{\Delta x}-\frac{f\left(u_{j+\frac{1}{2}}^{-}\right)-f\left(u_{j-\frac{1}{2}}^{+}\right)}{\Delta x}
$$

and

$$
\lim _{\Delta t \rightarrow 0} \bar{w}_{j+\frac{1}{2}}^{n+1}=\frac{a_{j+\frac{1}{2}}^{+} u_{j+\frac{1}{2}}^{+}-a_{j+\frac{1}{2}}^{-} u_{j+\frac{1}{2}}^{-}}{a_{j+\frac{1}{2}}^{+}-a_{j+\frac{1}{2}}^{-}}-\frac{f\left(u_{j+\frac{1}{2}}^{+}\right)-f\left(u_{j+\frac{1}{2}}^{-}\right)}{a_{j+\frac{1}{2}}^{+}-a_{j+\frac{1}{2}}^{-}}
$$

where $u_{j+\frac{1}{2}}^{+}=l_{j+1}\left(x_{j+\frac{1}{2}}\right)$ and $u_{j+\frac{1}{2}}^{-}=l_{j}\left(x_{j+\frac{1}{2}}\right)$ are the corresponding right and left values of the piecewise linear interpolant $\left\{l_{j}\right\}$, reconstructed at time $t$. A substitution of (3.2) and (3.3) in (3.1) results in a family of semi-discrete central-upwind schemes, which can be written in the conservative form,

$$
\frac{\mathrm{d}}{\mathrm{d} t} \bar{u}_{j}(t)=-\frac{H_{j+\frac{1}{2}}(t)-H_{j-\frac{1}{2}}(t)}{\Delta x}
$$


with numerical flux $H_{j+\frac{1}{2}}$, given by

$$
\begin{aligned}
H_{j+\frac{1}{2}}(t):= & \frac{a_{j+\frac{1}{2}}^{+} f\left(u_{j+\frac{1}{2}}^{-}\right)-a_{j+\frac{1}{2}}^{-} f\left(u_{j+\frac{1}{2}}^{+}\right)}{a_{j+\frac{1}{2}}^{+}-a_{j+\frac{1}{2}}^{-}}+\frac{a_{j+\frac{1}{2}}^{+} a_{j+\frac{1}{2}}^{-}}{a_{j+\frac{1}{2}}^{+}-a_{j+\frac{1}{2}}^{-}}\left[u_{j+\frac{1}{2}}^{+}-u_{j+\frac{1}{2}}^{-}\right] \\
& -\frac{a_{j+\frac{1}{2}}^{+} a_{j+\frac{1}{2}}^{-}}{2} \lim _{\Delta t \rightarrow 0}\left(\Delta t s_{j+\frac{1}{2}}^{n+1}\right) .
\end{aligned}
$$

If the slopes $\left\{s_{j+\frac{1}{2}}^{n+1}\right\}$ are chosen to be uniformly bounded independently of $\Delta t$, as in (2.10), then

$$
\lim _{\Delta t \rightarrow 0}\left(\Delta t s_{j+\frac{1}{2}}^{n+1}\right)=0
$$

and we obtain the semi-discrete scheme from [13]. A choice of $\left\{s_{j+\frac{1}{2}}^{n+1}\right\}$, for which

$$
q_{j+\frac{1}{2}}:=\lim _{\Delta t \rightarrow 0}\left(\Delta t s_{j+\frac{1}{2}}^{n+1}\right) \neq 0,
$$

results in the semi-discrete scheme (3.4) with a less dissipative numerical flux

$$
H_{j+\frac{1}{2}}(t)=\frac{a_{j+\frac{1}{2}}^{+} f\left(u_{j+\frac{1}{2}}^{-}\right)-a_{j+\frac{1}{2}}^{-} f\left(u_{j+\frac{1}{2}}^{+}\right)}{a_{j+\frac{1}{2}}^{+}-a_{j+\frac{1}{2}}^{-}}+a_{j+\frac{1}{2}}^{+} a_{j+\frac{1}{2}}^{-}\left[\frac{u_{j+\frac{1}{2}}^{+}-u_{j+\frac{1}{2}}^{-}}{a_{j+\frac{1}{2}}^{+}-a_{j+\frac{1}{2}}^{-}}-\frac{q_{j+\frac{1}{2}}}{2}\right] .
$$

Remarks.

1. If the $q_{j+\frac{1}{2}}$ 's are computed component-wise, then the scheme (3.4)-(3.5) is a Godunov-type central scheme, since it is based on integration over Riemann fans and does not require characteristic decomposition.

2. In the next section, we use a certain (partial) characteristic decomposition to obtain a semi-discrete scheme (3.4), (3.7) with nonzero $q_{j+\frac{1}{2}}$ 's. This will give a stable semi-discrete scheme, which will be less dissipative than the scheme (3.4)-(3.6), and will provide a higher resolution of contact waves.

3. Any stable ODE solver of an appropriate order can be used for the time discretization of the semi-discrete scheme (3.4)-(3.5). In the numerical examples below, we have used the second-order modified Euler method.

4. Instead of (2.1), one may use a higher-order non-oscillatory piecewise polynomial reconstruction, which will result in a higher-order semi-discrete scheme (for details, consult [13]).

\section{Partial CharaCteristic DeCOMPOSITION}

As we have already mentioned, the fully-discrete scheme (2.2)-(2.7), (2.9) and the semi-discrete scheme (3.4)(3.5) have a degree of freedom in choosing the slopes $\left\{s_{j+\frac{1}{2}}^{n+1}\right\}$ in (2.9) and (3.5), respectively. A possible choice of these slopes is (2.10). In this case, $s_{j+\frac{1}{2}}^{n+1} \sim \Delta w / \Delta x$, which leads to a scheme with a relatively large numerical dissipation, which can be decreased by choosing $s_{j+\frac{1}{2}}^{n+1}$ to be proportional to $\Delta w / \Delta t$. In the fully-discrete setting we may proceed, for example, as follows. First, we approximate the values of the solution at the points $x_{j+\frac{1}{2}, l}^{n}$ and $x_{j+\frac{1}{2}, r}^{n}$ at time level $t=t^{n+1}$. Since the solution is smooth there, we may use the Taylor expansion, and similarly to $(2.7)$ we obtain

$$
u_{j+\frac{1}{2}, l}^{n+1}:=u_{j+\frac{1}{2}, l}^{n}-\Delta t f\left(u_{j+\frac{1}{2}, l}^{n}\right)_{x}, \quad u_{j+\frac{1}{2}, r}^{n+1}:=u_{j+\frac{1}{2}, r}^{n}-\Delta t f\left(u_{j+\frac{1}{2}, r}^{n}\right)_{x} .
$$


We then apply the minmod limiter to these point values and $\bar{w}_{j+\frac{1}{2}}^{n+1}$ to compute the slopes $s_{j+\frac{1}{2}}^{n+1}$, namely

$$
s_{j+\frac{1}{2}}^{n+1}=\operatorname{minmod}\left(\theta \frac{\bar{w}_{j+\frac{1}{2}}^{n+1}-u_{j+\frac{1}{2}, l}^{n+1}}{\delta}, \frac{u_{j+\frac{1}{2}, r}^{n+1}-u_{j+\frac{1}{2}, l}^{n+1}}{2 \delta}, \theta \frac{u_{j+\frac{1}{2}, r}^{n+1}-\bar{w}_{j+\frac{1}{2}}^{n+1}}{\delta}\right), \quad \delta=\frac{\Delta t}{2}\left(a_{j+\frac{1}{2}}^{+}-a_{j+\frac{1}{2}}^{-}\right),
$$

and this completes the construction of a fully-discrete scheme (2.2)-(2.7), (2.9), (4.2).

The corresponding semi-discrete scheme is obtained by substituting (4.2) in (3.5). The limit is

$$
\lim _{\Delta t \rightarrow 0}\left(\Delta t s_{j+\frac{1}{2}}^{n+1}\right)=\frac{u_{j+\frac{1}{2}}^{+}-u_{j+\frac{1}{2}}^{-}}{a_{j+\frac{1}{2}}^{+}-a_{j+\frac{1}{2}}^{-}}
$$

and the resulting numerical flux is

$$
H_{j+\frac{1}{2}}(t):=\frac{a_{j+\frac{1}{2}}^{+} f\left(u_{j+\frac{1}{2}}^{-}\right)-a_{j+\frac{1}{2}}^{-} f\left(u_{j+\frac{1}{2}}^{+}\right)}{a_{j+\frac{1}{2}}^{+}-a_{j+\frac{1}{2}}^{-}}+\frac{a_{j+\frac{1}{2}}^{+} a_{j+\frac{1}{2}}^{-}}{2}\left[\frac{u_{j+\frac{1}{2}}^{+}-u_{j+\frac{1}{2}}^{-}}{a_{j+\frac{1}{2}}^{+}-a_{j+\frac{1}{2}}^{-}}\right] .
$$

Obviously, the scheme (3.4), (4.3) has less dissipation than the central-upwind scheme (3.4)-(3.6), but it may have an oscillatory behavior. This instability is illustrated in numerical Example 3, Figures 5.6-5.7. However, it is possible to retain the stability of the nonlinear fields, and yet improve the resolution of contact discontinuities. It can be done if one applies the latter approach (the less dissipative reconstruction) only to the linear field(s).

To this end, we implement the idea of partial characteristic decomposition, introduced in [22] in the context of the one-dimensional Euler equations of gas dynamics,

$$
\frac{\partial}{\partial t}\left[\begin{array}{l}
\rho \\
m \\
E
\end{array}\right]+\frac{\partial}{\partial x}\left[\begin{array}{c}
m \\
\rho u^{2}+p \\
u(E+p)
\end{array}\right]=0, \quad p=(\gamma-1) \cdot\left(E-\frac{\rho}{2} u^{2}\right) .
$$

Here, $\rho, u, m=\rho u, p$ and $E$ are density, velocity, momentum, pressure and the total energy, respectively.

Let us recall that the corresponding Roe matrix $\hat{A}_{j+\frac{1}{2}}=\hat{A}\left(v_{j}, v_{j+1}\right)$ for (4.4) has eigenvalues

$$
\hat{a}_{j+\frac{1}{2}, 1}=\hat{u}_{j+\frac{1}{2}}-\hat{c}_{j+\frac{1}{2}}, \quad \hat{a}_{j+\frac{1}{2}, 2}=\hat{u}_{j+\frac{1}{2}}, \quad \hat{a}_{j+\frac{1}{2}, 3}=\hat{u}_{j+\frac{1}{2}}+\hat{c}_{j+\frac{1}{2}}
$$

and right eigenvectors

$$
\hat{R}_{j+\frac{1}{2}, 1}=\left[\begin{array}{c}
1 \\
\hat{u}-\hat{c} \\
\hat{H}-\hat{u} \hat{c}
\end{array}\right]_{j+\frac{1}{2}}, \quad \hat{R}_{j+\frac{1}{2}, 2}=\left[\begin{array}{c}
1 \\
\hat{u} \\
\frac{1}{2} \hat{u}^{2}
\end{array}\right]_{j+\frac{1}{2}}, \quad \hat{R}_{j+\frac{1}{2}, 3}=\left[\begin{array}{c}
1 \\
\hat{u}+\hat{c} \\
\hat{H}+\hat{u} \hat{c}
\end{array}\right]_{j+\frac{1}{2}} .
$$

The above quantities $\hat{u}, \hat{H}$ and $\hat{c}$ are defined by

$$
\hat{u}=\frac{\langle\sqrt{\rho} u\rangle}{\langle\sqrt{\rho}\rangle}, \quad \hat{H}=\frac{\langle\sqrt{\rho} H\rangle}{\langle\sqrt{\rho}\rangle}, \quad \hat{c}=\sqrt{(\gamma-1)\left(\hat{H}-\frac{1}{2} \hat{u}^{2}\right)},
$$

where $\langle v\rangle:=\frac{1}{2}\left(v_{j}+v_{j+1}\right)$ stands for the Roe average of $v_{j}$ and $v_{j+1}$. 
Further, we introduce the notation

$$
\begin{gathered}
\Delta^{r} v_{j+\frac{1}{2}}:=v_{j+\frac{1}{2}, r}-v_{j+\frac{1}{2}}, \quad \Delta^{l} v_{j+\frac{1}{2}}:=v_{j+\frac{1}{2}}-v_{j+\frac{1}{2}, l}, \\
\langle v\rangle^{r}:=\frac{1}{2}\left(v_{j+\frac{1}{2}, r}+v_{j+\frac{1}{2}}\right), \quad\langle v\rangle^{l}:=\frac{1}{2}\left(v_{j+\frac{1}{2}}+v_{j+\frac{1}{2}, l}\right),
\end{gathered}
$$

and $\hat{u}^{r}, \hat{H}^{r}, \hat{c}^{r}\left(\hat{u}^{l}, \hat{H}^{l}, \hat{c}^{l}\right)$, where the upper index $r(l)$ means that in formula $(4.7)\langle\cdot\rangle^{r}\left(\langle\cdot\rangle^{l}\right)$ is used instead of $\langle\cdot\rangle$. We now consider the partial characteristic decomposition

$$
u_{j+\frac{1}{2}, r}^{n+1}-\bar{w}_{j+\frac{1}{2}}^{n+1}=\Delta^{r} \widetilde{w}_{j+\frac{1}{2}}+\alpha_{j+\frac{1}{2}, 2}^{r}\left[\begin{array}{c}
1 \\
\hat{u}^{r} \\
\frac{1}{2}\left(\hat{u}^{r}\right)^{2}
\end{array}\right]_{j+\frac{1}{2}}:=\left[\begin{array}{c}
\Delta^{r} \widetilde{\rho} \\
\Delta^{r} \widetilde{m} \\
\Delta^{r} \widetilde{E}
\end{array}\right]_{j+\frac{1}{2}}+\alpha_{j+\frac{1}{2}, 2}^{r}\left[\begin{array}{c}
1 \\
\hat{u}^{r} \\
\frac{1}{2}\left(\hat{u}^{r}\right)^{2}
\end{array}\right]_{j+\frac{1}{2}}
$$

where

$$
\alpha_{j+\frac{1}{2}, 2}^{r}=\Delta^{r} \rho_{j+\frac{1}{2}}-\frac{\Delta^{r} p_{j+\frac{1}{2}}}{\left(\hat{c}_{j+\frac{1}{2}}^{r}\right)^{2}} .
$$

The difference $\bar{w}_{j+\frac{1}{2}}^{n+1}-u_{j+\frac{1}{2}, l}^{n+1}$ can be written in a similar form, namely

$$
\bar{w}_{j+\frac{1}{2}}^{n+1}-u_{j+\frac{1}{2}, l}^{n+1}=\left[\begin{array}{c}
\Delta^{l} \widetilde{\rho} \\
\Delta^{l} \widetilde{m} \\
\Delta^{l} \widetilde{E}
\end{array}\right]_{j+\frac{1}{2}}+\alpha_{j+\frac{1}{2}, 2}^{l}\left[\begin{array}{c}
1 \\
\hat{u}^{l} \\
\frac{1}{2}\left(\hat{u}^{l}\right)^{2}
\end{array}\right]_{j+\frac{1}{2}}, \quad \alpha_{j+\frac{1}{2}, 2}^{l}=\Delta^{l} \rho_{j+\frac{1}{2}}-\frac{\Delta^{l} p_{j+\frac{1}{2}}}{\left(\hat{c}_{j+\frac{1}{2}}^{l}\right)^{2}}
$$

We call (4.8), (4.9) a partial characteristic decomposition, since it isolates the characteristic projection on the contact field, and allows component-wise treatment of the other two fields. This enables us to treat linear and nonlinear fields separately and therefore it provides an additional degree of freedom - we may use different limiters for the different fields. It allows us to improve the resolution of contact waves without affecting the stability of shocks and rarefactions.

Next, we employ the partial characteristic decomposition and use, for example, the slopes (2.10) for the nonlinear fields, and a less dissipative reconstruction for the linear field. The performed numerical experiments show that it is even possible to avoid the application of nonlinear limiters to the linear field, namely we can choose the following slopes $s_{j+\frac{1}{2}}^{n+1}$,

$$
\begin{aligned}
& s_{j+\frac{1}{2}}^{n+1}=2 \cdot \operatorname{minmod}\left(\theta \frac{\Delta^{l} \widetilde{w}_{j+\frac{1}{2}}}{x_{j+\frac{1}{2}, r}^{n}-x_{j-\frac{1}{2}, r}^{n}}, \frac{\Delta \widetilde{w}_{j}}{x_{j+\frac{3}{2}, l}^{n}+x_{j+\frac{1}{2}, r}^{n}-x_{j+\frac{1}{2}, l}^{n}-x_{j-\frac{1}{2}, r}^{n}}, \theta \frac{\Delta^{r} \widetilde{w}_{j+\frac{1}{2}}}{x_{j+\frac{3}{2}, l}^{n}-x_{j+\frac{1}{2}, l}^{n}}\right) \\
& +\frac{1}{\Delta t\left(a_{j+\frac{1}{2}}^{+}-a_{j+\frac{1}{2}}^{-}\right)}\left\{\alpha_{j+\frac{1}{2}, 2}^{r}\left[\begin{array}{c}
1 \\
\hat{u}^{r} \\
\frac{1}{2}\left(\hat{u}^{r}\right)^{2}
\end{array}\right]_{j+\frac{1}{2}}+\alpha_{j+\frac{1}{2}, 2}^{l}\left[\begin{array}{c}
1 \\
\hat{u}^{l} \\
\frac{1}{2}\left(\hat{u}^{l}\right)^{2}
\end{array}\right]_{j+\frac{1}{2}}\right\} \text {, }
\end{aligned}
$$

which, together with (2.2)-(2.7), (2.9), completes our fully-discrete scheme for the Euler equations (4.4).

Substitution of (4.10) in (3.5) results in the semi-discrete scheme (3.4), (3.7) with

$$
q_{j+\frac{1}{2}}=\frac{\alpha_{j+\frac{1}{2}, 2}}{a_{j+\frac{1}{2}}^{+}-a_{j+\frac{1}{2}}^{-}}\left[\begin{array}{c}
1 \\
\hat{u} \\
\frac{1}{2} \hat{u}^{2}
\end{array}\right]_{j+\frac{1}{2}} .
$$


Here, $\alpha_{j+\frac{1}{2}, 2}$ are the coefficients of the partial characteristic decomposition of

$$
u_{j+\frac{1}{2}}^{+}-u_{j+\frac{1}{2}}^{-}=\left[\begin{array}{c}
\widetilde{\rho}_{j+\frac{1}{2}}^{+}-\widetilde{\rho}_{j+\frac{1}{2}}^{-} \\
\widetilde{m}_{j+\frac{1}{2}}^{+}-\widetilde{m}_{j+\frac{1}{2}}^{-} \\
\widetilde{E}_{j+\frac{1}{2}}^{+}-\widetilde{E}_{j+\frac{1}{2}}^{-}
\end{array}\right]+\alpha_{j+\frac{1}{2}, 2}\left[\begin{array}{c}
1 \\
\hat{u} \\
\frac{1}{2} \hat{u}^{2}
\end{array}\right]_{j+\frac{1}{2}},
$$

which corresponds to a Roe matrix $\hat{A}\left(u_{j+\frac{1}{2}}^{+}, u_{j+\frac{1}{2}}^{-}\right)$.

Remarks.

1. In practice one may avoid the computation of $u_{j+\frac{1}{2}, r}^{n+1}$ and $u_{j+\frac{1}{2}, l}^{n+1}$ in (4.1) by replacing them in (4.8) and (4.9) with $\bar{w}_{j+1}^{n+1}$ and $\bar{w}_{j}^{n+1}$, respectively. This does not effect the overall accuracy of the fully-discrete scheme. Moreover, the performed numerical experiments illustrate an improvement of the resolution of contact discontinuities.

2. The same approach of partial characteristic decomposition can be used for any other strictly hyperbolic system with a known Roe matrix and at least one linear field.

3 . If we set $u_{j+\frac{1}{2}}^{+}=\bar{u}_{j+1}$ and $u_{j+\frac{1}{2}}^{-}=\bar{u}_{j}$ in (3.7) and (4.12) (which is equivalent to setting $s_{j}^{n}=0$ in (2.1)), the resulting first-order scheme (3.4), (3.7), (4.11)-(4.12) is similar to the first-order scheme from [4].

4. Notice that in contrast to the NT scheme (introduced in [22]), where the partial characteristic decomposition is used both at the reconstruction and the evolution steps, the proposed schemes use it only for the computation of the slopes $s_{j+\frac{1}{2}}^{n+1}$. Therefore, the use of the partial characteristic decomposition in the presented schemes is computationally nonexpensive.

\section{NUMERICAL EXAMPLES}

In this section, we study the performance of the introduced fully-discrete (FD) scheme (2.2)-(2.7), (2.9), (4.10) and the semi-discrete (modified SD) scheme (3.4), (3.7), (4.11)-(4.12). They are tested on the onedimensional Euler equations (4.4) with $\gamma=1.4$, subject to different initial conditions, and are compared to the original NT scheme, the NT scheme that utilizes the partial characteristic decomposition (according to the recipe (4.19) and (4.21) in [22]), and with the semi-discrete (SD) scheme, defined in (3.4), (4.3). We use the CFL number 0.475 and $\theta=1.3$ (in the minmod limiter (2.2)) in all computations.

As expected, the numerical examples below clearly demonstrate that the FD and modified SD schemes give a better resolution of the contact waves.

\section{Example 1: Steady contact discontinuity}

We solve the system (4.4) on the interval $[-0.2,0.2]$, subject to the initial data

$$
\vec{u}(x, 0)= \begin{cases}\vec{u}_{L}=(1,0,2.5)^{T}, & x<0, \\ \vec{u}_{R}=(0.5,0,2.5)^{T}, & x>0,\end{cases}
$$

which corresponds to a single steady contact discontinuity. We use 80 grid points and compute the solution at a final time $T=10$. Figure 5.1 shows the density, computed using the NT, the NT scheme with partial decomposition, and the SD scheme. The excessive numerical viscosity, present in all these schemes, does not 


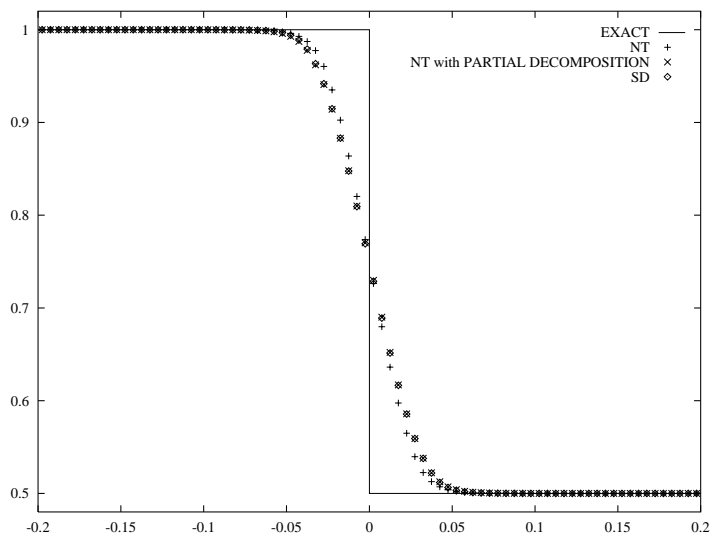

FIGURE 5.1. Steady contact - density.

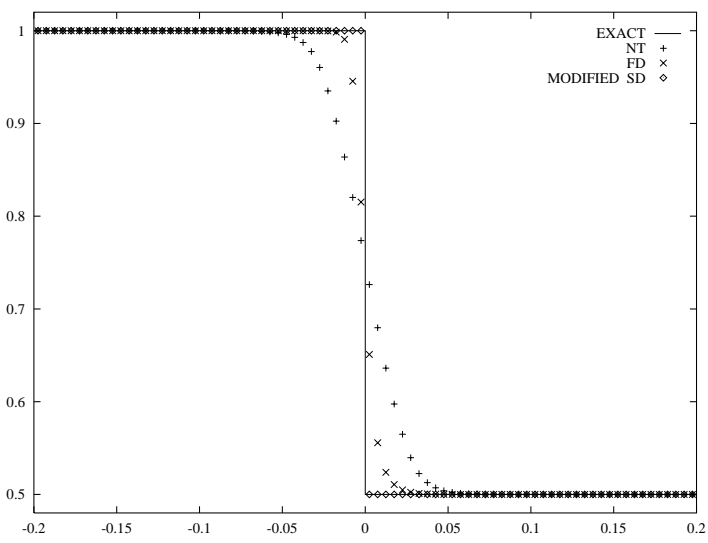

FIGURE 5.2. Steady contact - density.

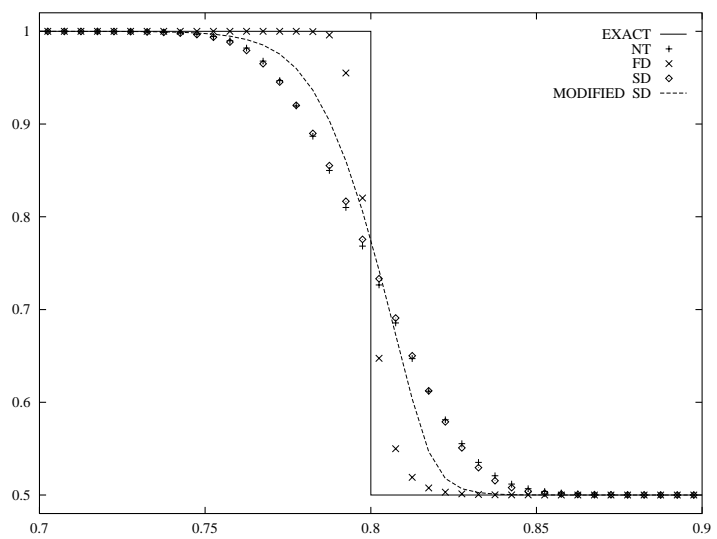

Figure 5.3. Moving contact - density; zoom at $[0.7,0.9]$.

allow a sharp resolution of the discontinuity. In contrast, the FD scheme gives a much better resolution, and the modified SD scheme provides a perfect resolution of the steady contact wave (see Fig. 5.2).

Remark. This simple example, and the ones that follow (see Figs. 5.4-5.7), demonstrate that the partial characteristic decomposition in the context of the NT scheme does not provide a big improvement, unless it is used together with the ACM (see $[20,22]$ ).

\section{Example 2: Moving contact discontinuity}

We consider problem (4.4) on the interval $[-0.1,0.9]$, subject to the initial data

$$
\vec{u}(x, 0)= \begin{cases}\vec{u}_{L}=(1,0.1,2.505)^{T}, & x<0 \\ \vec{u}_{R}=(0.5,0.05,2.5025)^{T}, & x>0\end{cases}
$$


which is an example of a single moving contact discontinuity. We compute the approximate solution at time $T=8$, using 200 grid points. The graph of the density is presented in Figure 5.3, and it clearly illustrates the advantages of the FD and the modified SD schemes in this example.

\section{Example 3: Lax problem}

The Riemann problem with initial data

$$
\vec{u}(x, 0)= \begin{cases}\vec{u}_{L}=(0.445,0.311,8.928)^{T}, & x<0, \\ \vec{u}_{R}=(0.5,0,1.4275)^{T}, & x>0,\end{cases}
$$

was proposed by Lax in [15]. Figures 5.4-5.7 show the approximate solution, computed with 200 grid points at time $T=0.16$ by the NT, the NT with partial decomposition and the SD schemes. The quality of the resolution, provided by these schemes, is about the same, but the SD scheme generates spurious oscillations in the pressure and velocity fields, Figures 5.6-5.7. As mentioned in Section 4, this is due to the insufficient numerical dissipation, present in the SD scheme. Notice that the oscillations occur around the location of the contact discontinuity.

In Figures 5.8-5.10 we compare the SD scheme with the modified SD scheme, where a low dissipative correction is applied only to the linear field. No oscillations are observed in this case.

The solution, computed by the FD scheme, is presented in Figures 5.11-5.14. Compared with the modified SD scheme, our fully-discrete scheme provides much better resolution of the contact wave. This can be clearly seen in Figure 5.12 - the modified SD scheme has twice as many points in the numerical contact layer as the FD scheme has.

\section{Example 4: Woodward-Colella problem}

Finally, we solve the system (4.4) on the interval $[0,1]$ with the initial data

$$
\vec{u}(x, 0)= \begin{cases}\vec{u}_{L}=(1,0,2500)^{T}, & 0 \leq x<0.1, \\ \vec{u}_{M}=(1,0,0.025)^{T}, & 0.1 \leq x<0.9, \\ \vec{u}_{R}=(1,0,250)^{T}, & 0.9 \leq x<1,\end{cases}
$$

and solid wall boundary conditions, applied to both ends. The example describes the interaction of blast waves and was proposed by Woodward and Colella in [26].

We compute the solution at time levels $T=0.01,0.03$ and 0.038 , using $N=400$ grid points. A reference solution is computed by the FD scheme with $N=3200$ grid points and the least oscillatory minmod limiter (2.2) with $\theta=1$.

Figures 5.15-5.16, 5.19-5.20 and 5.23-5.24 show that the SD and the modified SD schemes provide slightly better resolution than the NT scheme. This can be seen in Figures 5.16, 5.20 and 5.24, where the values at the peaks are compared. One can also observe that all these schemes poorly resolve the contact discontinuities. The fully-discrete (FD) scheme cannot resolve them completely as well, but at least it indicates the presence of contact waves. At the same time, the other tested central schemes fail to give even a qualitative behavior of the solution near the contact fields. This demonstrates the advantage of the FD scheme, as it can be seen in Figures 5.17-5.18, 5.21-5.22, 5.25-5.26. Moreover, the FD scheme also offers a better overall resolution (compare the values at the peaks). 


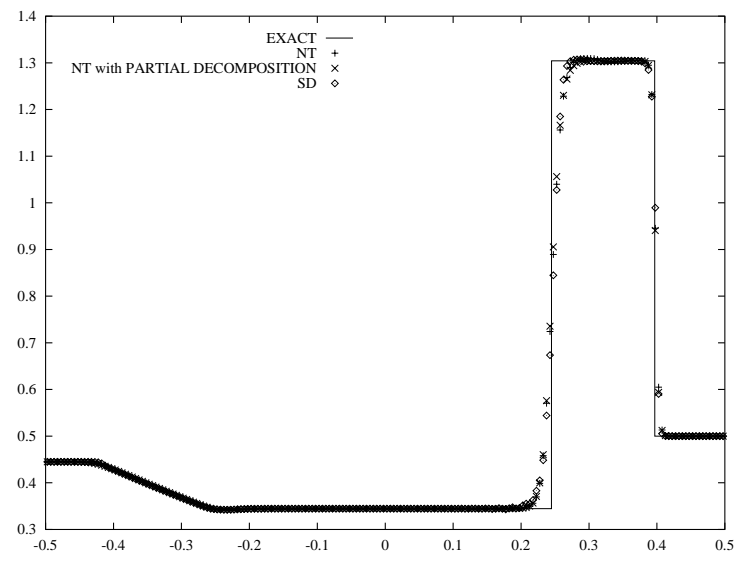

FiguRE 5.4. Lax problem - density.

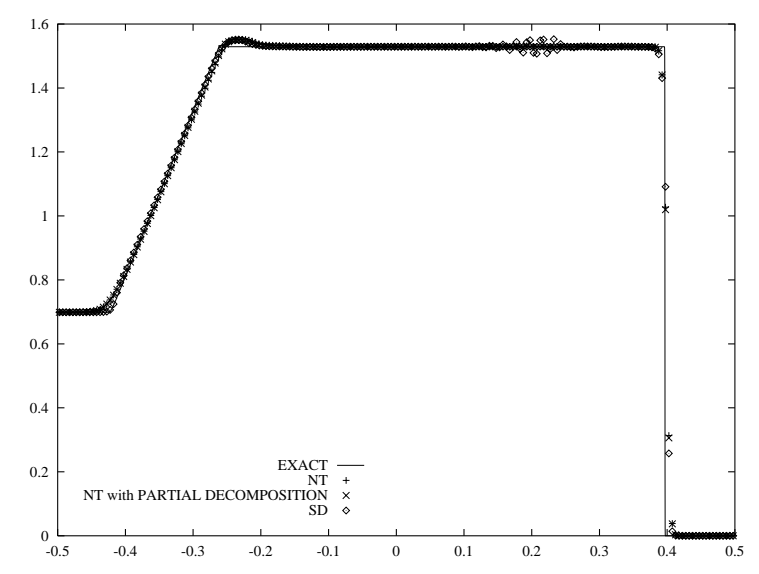

Figure 5.6. Lax problem - velocity.

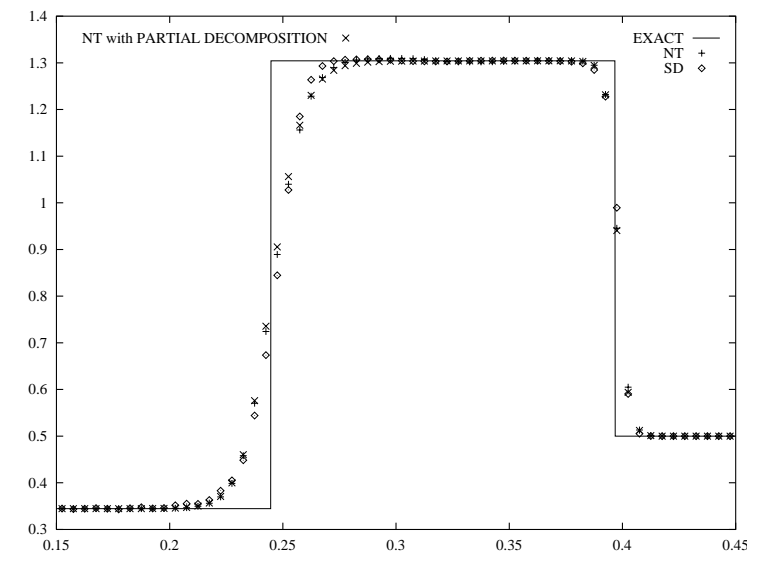

Figure 5.5. Zoom at $[0.15,0.45]$.

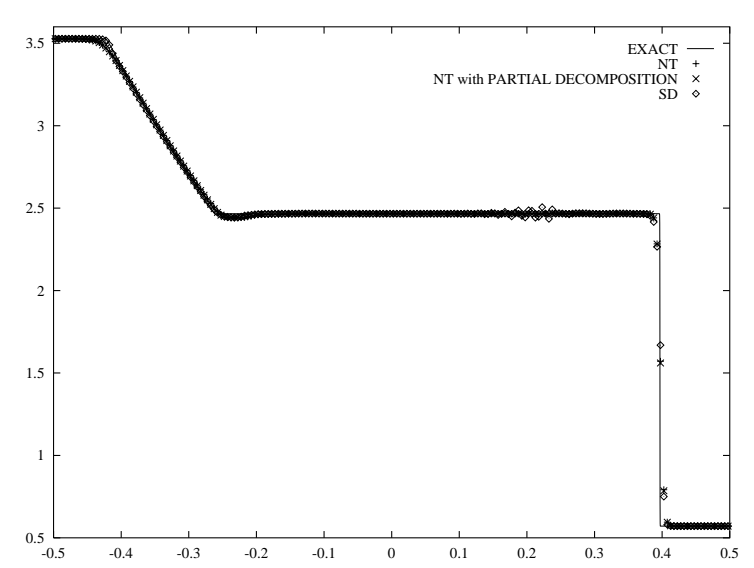

FiguRE 5.7. Lax problem - pressure.

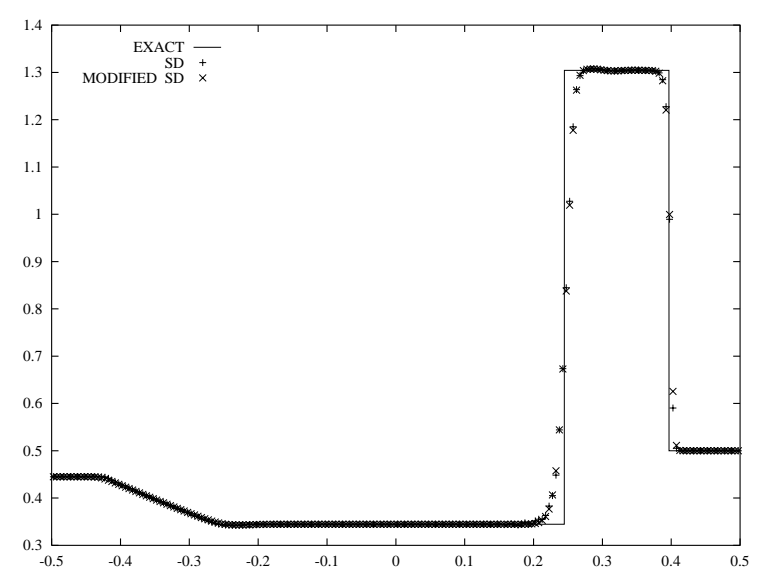

FiguRE 5.8. Lax problem - density. 


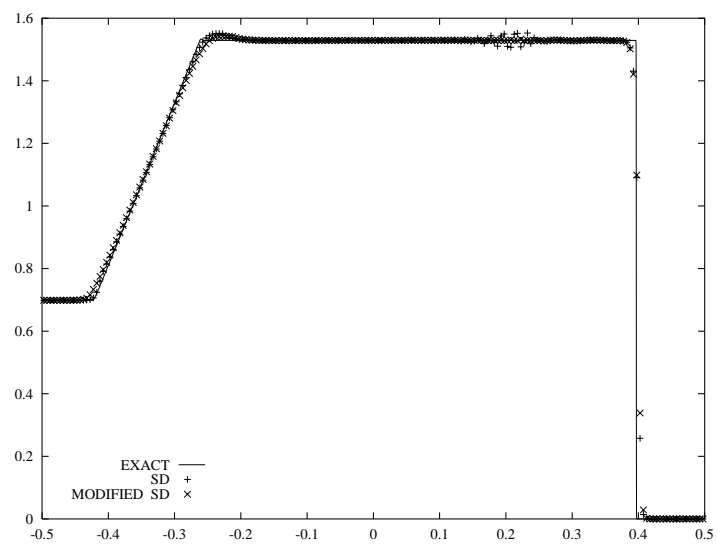

FIGURE 5.9. Lax problem - velocity.

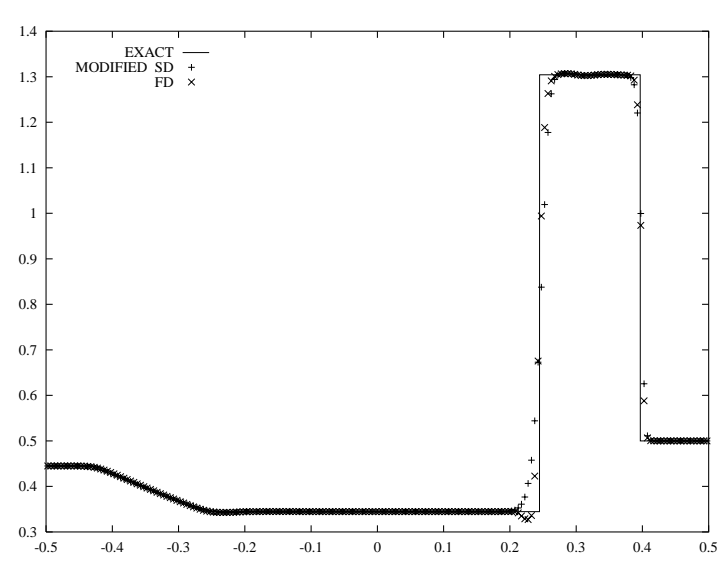

FiguRE 5.11. Lax problem - density.

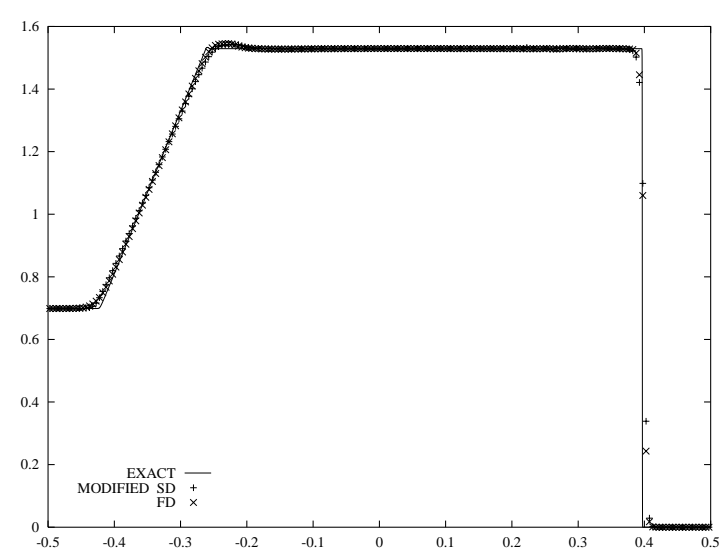

FiguRE 5.13. Lax problem - velocity.

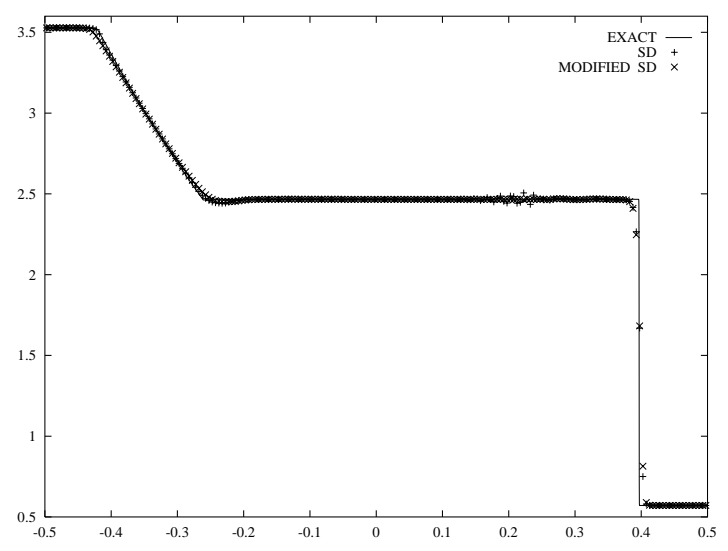

FiguRE 5.10. Lax problem - pressure.

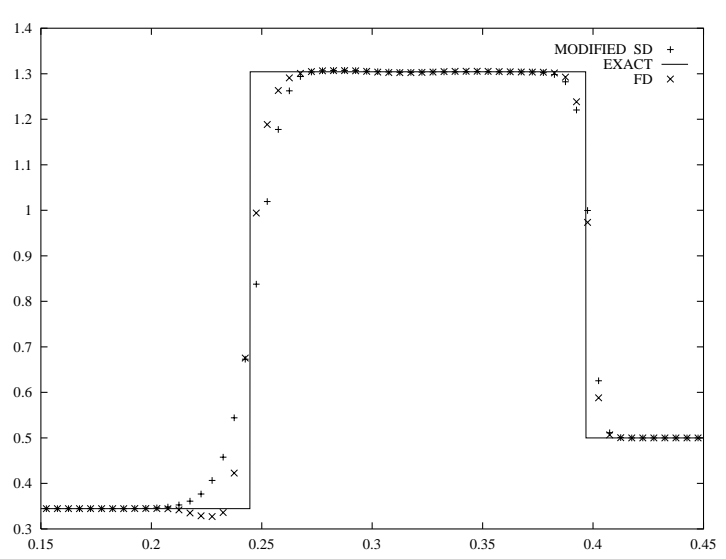

Figure 5.12. Zoom at $[0.15,0.45]$.

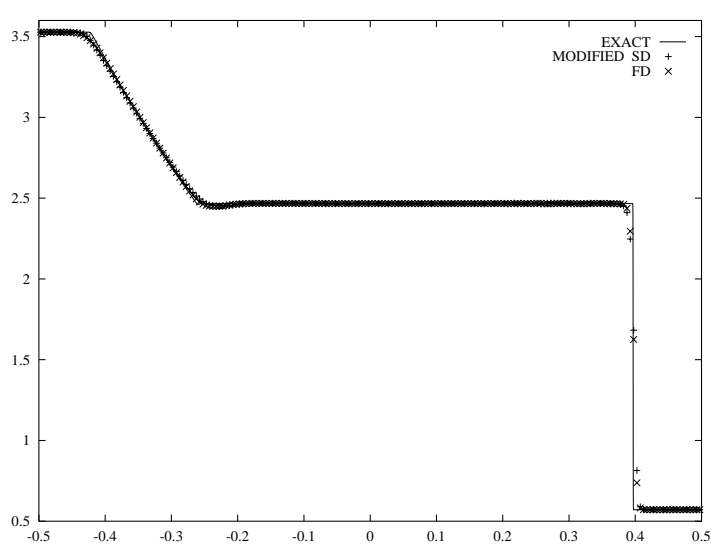

FiguRE 5.14. Lax problem - pressure. 


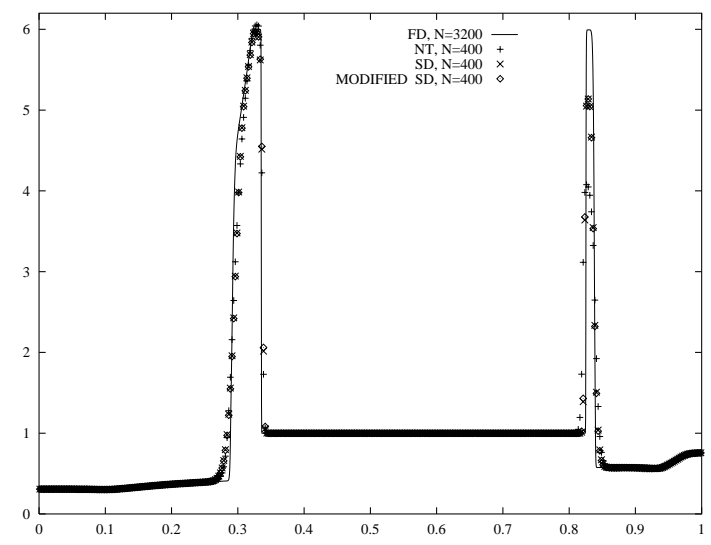

Figure 5.15. WC problem - density, $\mathbf{T}=\mathbf{0 . 0 1}$.

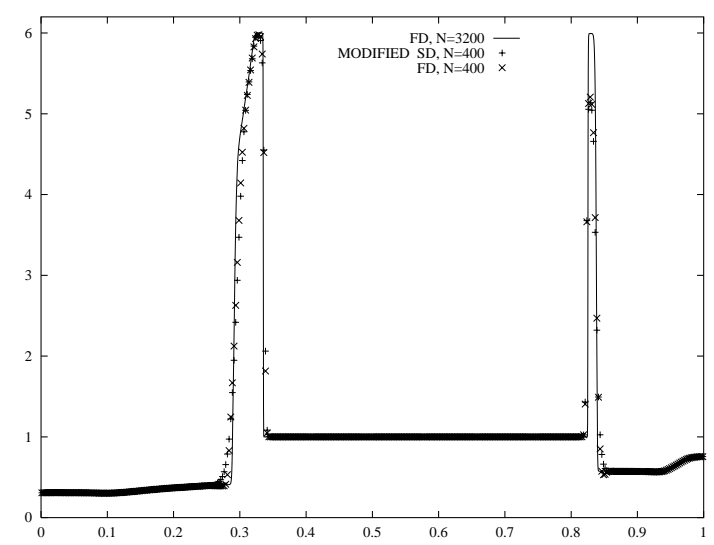

Figure 5.17. WC problem - density, $\mathbf{T}=\mathbf{0 . 0 1}$.

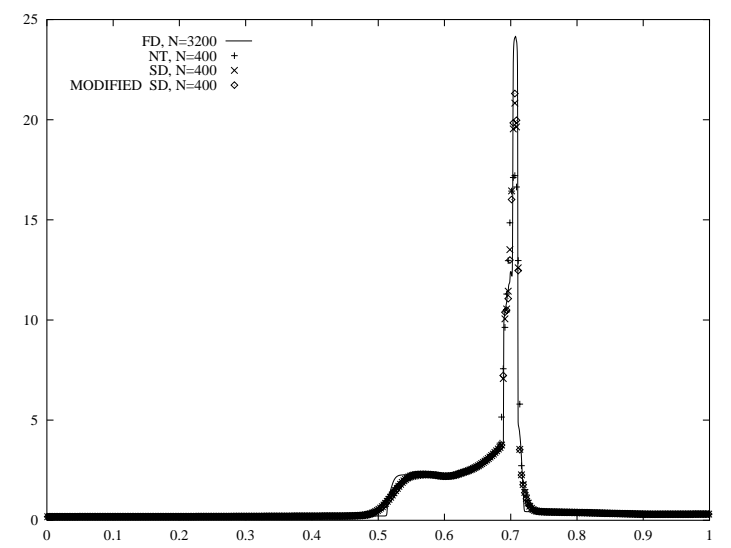

Figure 5.19. WC problem - density, $\mathbf{T}=\mathbf{0 . 0 3}$.

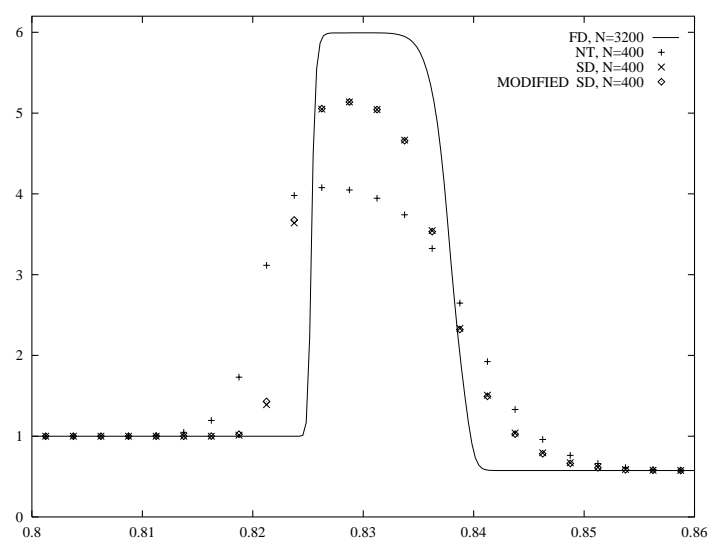

FigURE 5.16. Zoom at $[0.8,0.86]$.

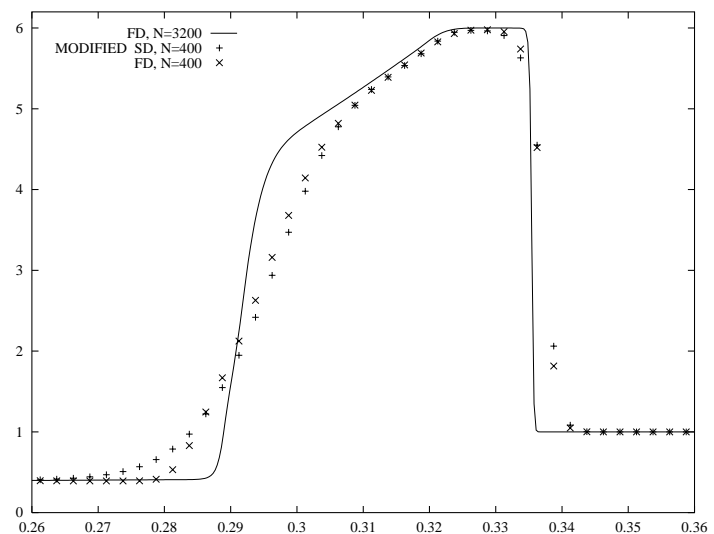

FiguRE 5.18. Zoom at $[0.26,0.36]$.

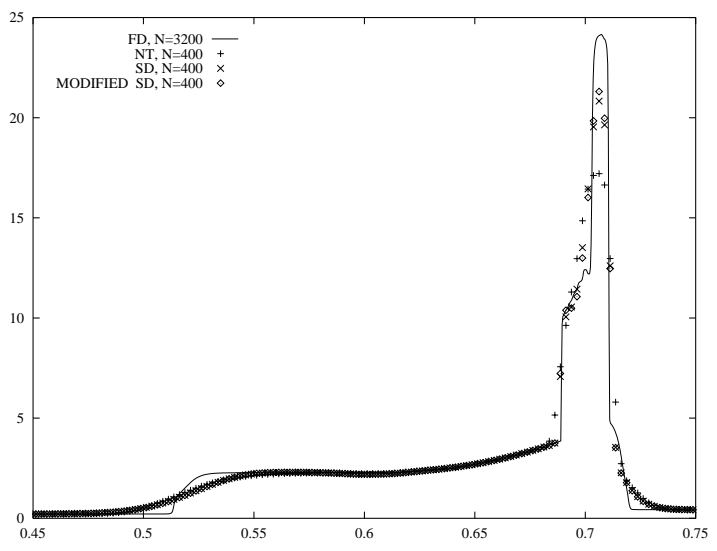

Figure 5.20. Zoom at $[0.45,0.75]$. 


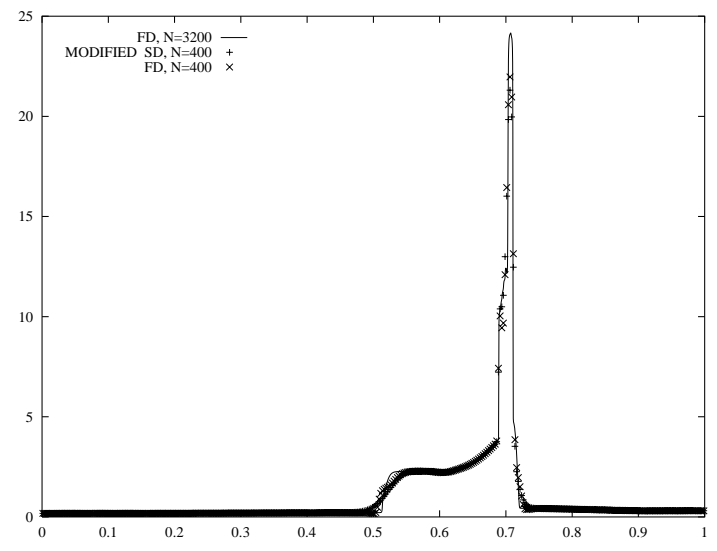

Figure 5.21. WC problem - density, $\mathbf{T}=\mathbf{0 . 0 3}$.

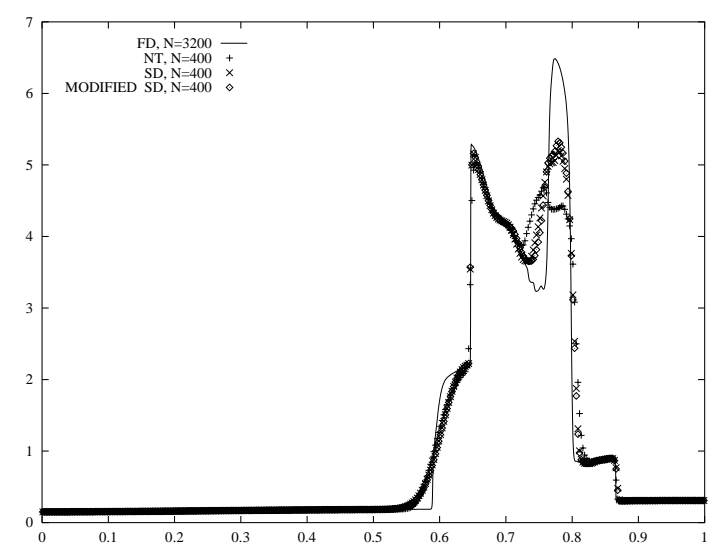

Figure 5.23. WC problem - density, $\mathbf{T}=\mathbf{0 . 0 3 8}$.

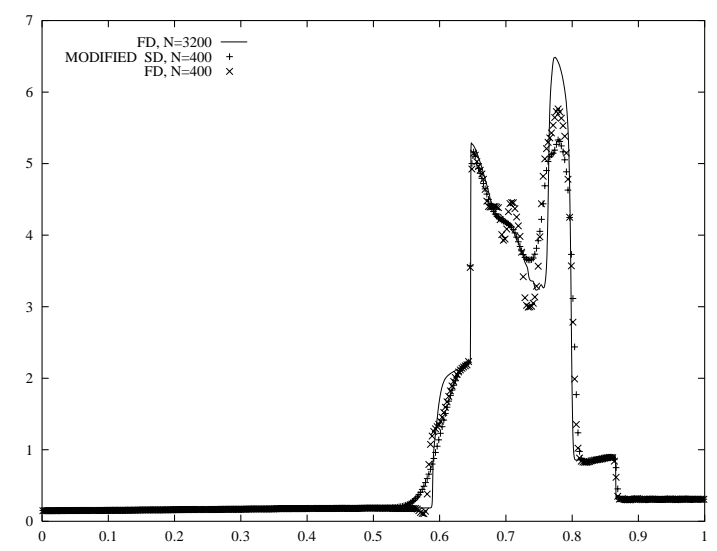

Figure 5.25. WC problem - density, $\mathbf{T}=\mathbf{0 . 0 3 8}$.

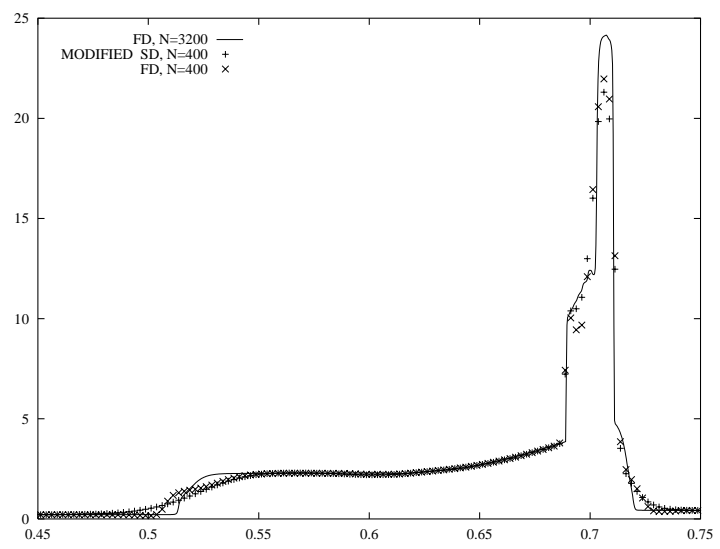

Figure 5.22. Zoom at $[0.45,0.75]$.

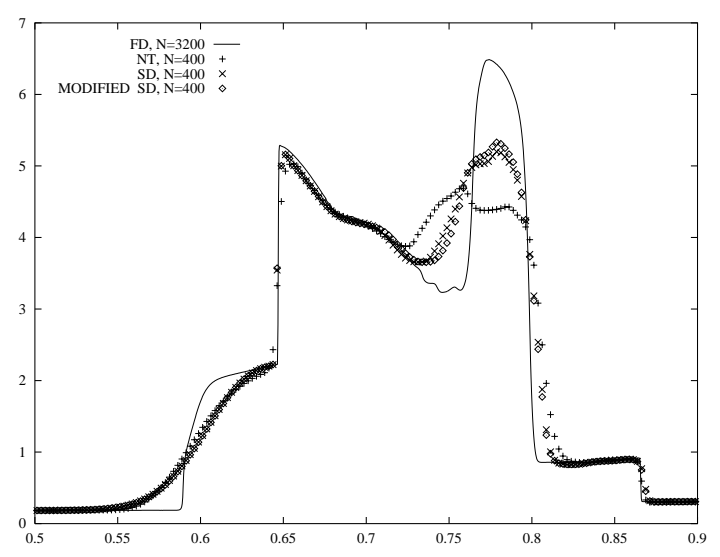

FIGURE 5.24. Zoom at $[0.5,0.9]$.

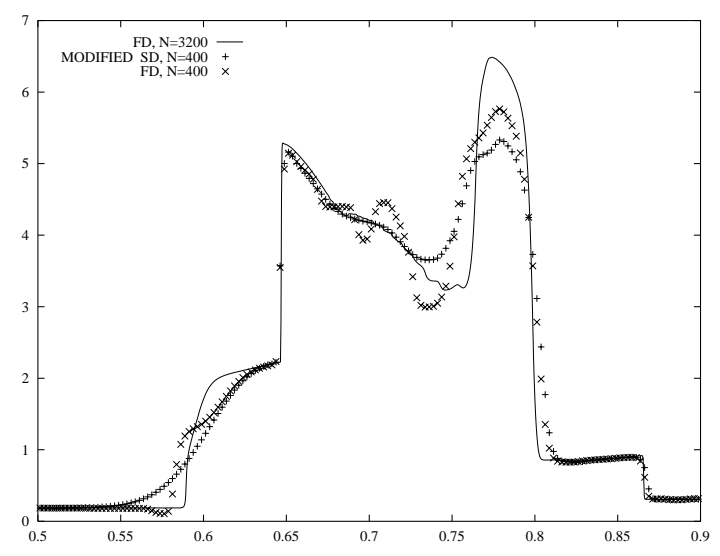

FiguRe 5.26. Zoom at $[0.5,0.9]$. 
Acknowledgements. We would like to thank Dr. S. Noelle for his helpful suggestions. The work of A. Kurganov was supported in part by the NSF Group Infrastructure Grant and the NSF Grant DMS-0073631.

\section{REFERENCES}

[1] P. Arminjon and M.-C. Viallon, Généralisation du schéma de Nessyahu-Tadmor pour une équation hyperbolique à deux dimensions d'espace. C.R. Acad. Sci. Paris Sér. I 320 (1995) 85-88.

[2] P. Arminjon, M.-C. Viallon and A. Madrane, A finite volume extension of the Lax-Friedrichs and Nessyahu-Tadmor schemes for conservation laws on unstructured grids. Int. J. Comput. Fluid Dyn. 9 (1997) 1-22.

[3] F. Bianco, G. Puppo and G. Russo, High order central schemes for hyperbolic systems of conservation laws. SIAM J. Sci. Comput. 21 (1999) 294-322.

[4] B. Einfeldt, On Godunov-type methods for gas dynamics. SIAM J. Numer. Anal. 25 (1988) 294-318.

[5] K.O. Friedrichs, Symmetric hyperbolic linear differential equations. Comm. Pure Appl. Math. 7 (1954) 345-392.

[6] A. Harten, The artificial compression method for computation of shocks and contact discontinuities. III. Self-adjusting hybrid schemes. Math. Comp. 32 (1978) 363-389.

[7] A. Harten, High resolution schemes for hyperbolic conservation laws. J. Comput. Phys. 49 (1983) 357-393.

[8] A. Harten, B. Engquist, S. Osher and S.R. Chakravarthy, Uniformly high order accurate essentially non-oscillatory schemes III. J. Comput. Phys. 71 (1987) 231-303.

[9] G.-S. Jiang and E. Tadmor, Non-oscillatory central schemes for multidimensional hyperbolic conservation laws. SIAM J. Sci. Comput. 19 (1998) 1892-1917.

[10] A. Kurganov, Conservation laws: stability of numerical approximations and nonlinear regularization. Ph.D. thesis, Tel-Aviv University, Israel (1997).

[11] A. Kurganov and D. Levy, A third-order semi-discrete central scheme for conservation laws and convection-diffusion equations. SIAM J. Sci. Comput. (to appear).

[12] A. Kurganov and G. Petrova, A third-order semi-discrete genuinely multidimensional central scheme for hyperbolic conservation laws and related problems. Numer. Math. (to appear).

[13] A. Kurganov, S. Noelle and G. Petrova, Semi-Discrete Central-Upwind Schemes for Hyperbolic Conservation Laws and Hamilton-Jacobi Equations. SIAM J. Sci. Comput. (submitted).

[14] A. Kurganov and E. Tadmor, New high-resolution central schemes for nonlinear conservation laws and convection-diffusion equations. J. Comput. Phys. 160 (2000) 241-282.

[15] P.D. Lax, Weak solutions of nonlinear hyperbolic equations and their numerical computation. Comm. Pure Appl. Math. 7 (1954) 159-193.

[16] B. van Leer, Towards the ultimate conservative difference scheme. V. A second order sequel to Godunov's method. J. Comput. Phys. 32 (1979) 101-136.

[17] D. Levy, G. Puppo and G. Russo, Central WENO schemes for hyperbolic systems of conservation laws. ESAIM: M2AN 33 (1999) $547-571$.

[18] D. Levy, G. Puppo and G. Russo, A third order central WENO scheme for 2D conservation laws. Appl. Numer. Math. 33 (2000) $407-414$.

[19] D. Levy, G. Puppo and G. Russo, Compact central WENO schemes for multidimensional conservation laws. SIAM J. Sci. Comput. 22 (2000) 656-672.

[20] K.-A. Lie and S. Noelle, Remarks on high-resolution non-oscillatory central schemes for multi-dimensional systems of conservation laws. Part I: An improved quadrature rule for the flux-computation. SIAM J. Sci. Comput. (submitted).

[21] X.-D. Liu and E. Tadmor, Third order nonoscillatory central scheme for hyperbolic conservation laws. Numer. Math. 79 (1998) 397-425.

[22] H. Nessyahu and E. Tadmor, Non-oscillatory central differencing for hyperbolic conservation laws. J. Comput. Phys. 87 (1990) 408-463.

[23] S. Osher and E. Tadmor, On the convergence of difference approximations to scalar conservation laws. Math. Comp. 50 (1988) 19-51.

[24] R. Sanders and A. Weiser, A high order staggered grid method for hyperbolic systems of conservation laws in one space dimension. Comput. Methods Appl. Mech. Engrg. 75 (1989) 91-107.

[25] R. Sanders and A. Weiser, High resolution staggered mesh approach for nonlinear hyperbolic systems of conservation laws. J. Comput. Phys. 101 (1992) 314-329.

[26] P. Woodward and P. Colella, The numerical solution of two-dimensional fluid flow with strong shocks. J. Comput. Phys. 54 (1988) $115-173$. 